\title{
Development of a Two-Dimensional Model for Predicting Transdermal Permeation with the Follicular Pathway: Demonstration with a Caffeine Study
}

\author{
Panayiotis Kattou ' - Guoping Lian ${ }^{1,2} \cdot$ Stephen Glavin ${ }^{2}$ • lan Sorrell ${ }^{2}$ Tao Chen ' (1)
}

Received: 7 April 2017 / Accepted: 12 June 2017 / Published online: 28 June 2017

(C) The Author(s) 2017. This article is an open access publication

\begin{abstract}
Purpose The development of a new two-dimensional (2D) model to predict follicular permeation, with integration into a recently reported multi-scale model of transdermal permeation is presented.
\end{abstract}

Methods The follicular pathway is modelled by diffusion in sebum. The mass transfer and partition properties of solutes in lipid, corneocytes, viable dermis, dermis and systemic circulation are calculated as reported previously [Pharm Res 33 (2016) 1602]. The mass transfer and partition properties in sebum are collected from existing literature. None of the model input parameters was fit to the clinical data with which the model prediction is compared.

Results The integrated model has been applied to predict the published clinical data of transdermal permeation of caffeine. The relative importance of the follicular pathway is analysed. Good agreement of the model prediction with the clinical data has been obtained. The simulation confirms that for caffeine the follicular route is important; the maximum bioavailable concentration of caffeine in systemic circulation with open hair follicles is predicted to be $20 \%$ higher than that when hair follicles are blocked.

Conclusions The follicular pathway contributes to not only short time fast penetration, but also the overall systemic bioavailability. With such in silico model, useful information can be obtained for caffeine disposition and localised delivery in lipid, corneocytes, viable dermis, dermis and the hair follicle. Such detailed information is difficult to obtain experimentally.

Tao Chen

t.chen@surrey.ac.uk

Department of Chemical and Process Engineering University of Surrey, Guildford GU2 7XH, UK

2 Unilever, Colworth Science Park, Sharnbrook, Bedfordshire MK44 ILQ, UK
KEY WORDS bioavailability · diffusion · in silico modelling . pharmacokinetic model · transdermal drug delivery

\section{ABBREVIATIONS \\ CVODE \\ C-language variable-coefficients ODE solver \\ $\mathrm{HF}$ \\ ODE \\ QSPR \\ SC \\ Hair follicle \\ SUNDIALS \\ Ordinary differential equation \\ Quantitative structure-property relationship Stratum corneum \\ equation solvers \\ VE $\quad$ Viable epidermis}

\section{INTRODUCTION}

A major challenge for scientific research regarding skin penetration of drugs, cosmetics, etc., is the development of robust non-animal methods to test percutaneous absorption and bioavailability. Dermal absorption due to exposure to agrochemicals and environmental pollutants is also becoming a global concern. Experimental approaches including in vivo, ex vivo, in vitro and clinical studies on human volunteers are often expensive and time consuming $(1,2)$. Additionally, there is a general trend in safety regulatory guidelines worldwide to move away from animal testing of cosmetic products and ingredients (e.g. the European Commission) (3). In parallel to the advancement in experimental methods, in silico modelling of dermal absorption and delivery has been demonstrated to be useful in refining and reducing the experiments needed, to enable faster design of new products and more reliable safety assessment, and to improve the understanding of the transport process (2). In addition, a mechanistic model can help analyse the relative importance of different penetration pathways; it can be used to examine the impact of physico-chemical properties of the chemical, the physiological variability and 
application scenarios on penetration (4). As a result, in silico modelling has become an important tool in the study of topical and transdermal delivery.

Within this context, quantitative structure-permeability relationship (QSPR) models emerged mainly focusing on estimating the permeability coefficient or maximum flux of the chemicals (5). The compartmental approach, which treats the skin layers as different units of uniform concentration (6), was the next step of in silico modelling. Then, the introduction of more sophisticated diffusion-based models has attracted substantial attention since its appearance. These models produce spatially explicit and time dependent predictions of transdermal permeation following topical exposure. The majority of early diffusion-based models do not consider the heterogeneity of the stratum corneum (6-10). When the bricks-andmortar structure of the stratum corneum was introduced and its importance acknowledged, the main challenge was to obtain the transport properties of chemical compounds in different domains of the skin. In some cases the transport properties are obtained by fitting to experimental skin penetration data $(11,12)$. In such cases the main problem is that the model only works well with that particular experimental dataset. This limits the range of chemicals the model can be used for prediction.

Subsequently, Wang et al. (13) created a predictive model of transdermal permeation that derived transport properties of the solute from fundamental principles. Chen et al. $(14,15)$ presented a multi-scale approach using a similar bricks-andmortar structure for the stratum corneum. These later modelling studies adopted a multi-scale approach where transport properties of skin lipids and cornecocytes are determined separately, e.g. through molecular modelling and QSPRs, achieving improved prediction accuracy. Later, Dancik et al. (16) and Chen et al. (17) further included viable epidermis and dermis. Some excellent review articles have been published to summarise recent progress in this area; see e.g. $(4,6,18)$. Recently we extended the model of Chen et al. (17) to include absorption into the systemic circulation and subsequent kinetics (19).

However, as far as the penetration pathways are concerned there is a noticeable gap with respect to the follicular pathway. In the past decade many studies (20-29) confirmed and highlighted the important contribution of the hair follicles to transdermal penetration. Although hair follicles occupy only ca. $0.1 \%$ of the skin surface, their diffusion coefficient can be orders of magnitude higher than that in the stratum corneum, and thus the overall effect can be significant (30). This pathway has been considered in some simple compartmental models $(21,31,32)$ which, however, have limited predictive capability because of the need for parameter fitting of the model to experimental data.

The aim of this study is to develop a mechanistic model of the follicular pathway and integrate the follicular pathway into our latest multi-scale model of transdermal permeation (19). The stratum corneum, viable epidermis and dermis as well as the systemic circulation kinetics are modelled using the same methodology as before $(14,17,19)$. The follicular pathway was integrated by considering the physiological and compositional properties of sebum and hair. The model's predictive capability is demonstrated through simulating an in vivo study of topical application of caffeine on skin with or without hair follicle blocking (22). Predicted caffeine plasma concentration-time profiles showed good agreement with the reported clinical data. A sensitivity analysis has been conducted to demonstrate the rational of the chosen sebum partition and transport properties of caffeine and the effect of variability in such properties on the overall transdermal delivery of caffeine.

\section{MATERIAL AND METHODS}

This section describes the method for modelling the follicular route of dermal absorption, and its integration with the previously reported multi-scale model of transdermal permeation and bioavailability (19). The technical details concerning the partition and transport properties of chemicals in skin and the blood circulation kinetics can be found in the appendices.

\section{The Hair Follicle Route: Modelling Approach}

The main focus of this study is on the follicular pathway. Although there exist debate with regard to the actual route of follicular penetration (20,29), studies have been conducted to examine at which phase of the hair growth cycle follicular penetration occurs. Domashenko et al. (33) reported a study on mouse skin and human scalp xenograft. They concluded that transfection of liposomes occurred only at the onset of a new growing stage of the hair cycle (33). In contrast another study, one that the assumptions of the current approach are based on, conducted on 8 human volunteers suggested that penetration through the shunt pathway only occurs when the hair follicle is active (27), where active hair follicle is characterised by hair growth and/or sebum production (34). It is also known that sebum is a penetrable medium (35-37) and that the diffusion coefficient in sebum $(35,36)$ is usually several orders of magnitude faster than that in hair (38). The above, alongside with the fact that hair it is a very dense material mainly made of keratin, support the argument that the sebum is the main transport route of follicular pathway. The gap between the hair infundibulum and the skin is assumed to be filled with sebum.

Based on the above assumption, the hair follicle anatomy (Fig. 1a) is converted into a 2D domain of heterogeneous material as illustrated in Fig. 1b, upon which a mathematical model is developed. Figure $1 \mathrm{~b}$ shows the different 
Fig. I Simplification of hair follicle anatomy (a) into a 2D modelling domain (b). Note that the modelling domain corresponds to the unshaded area in (a). a

b

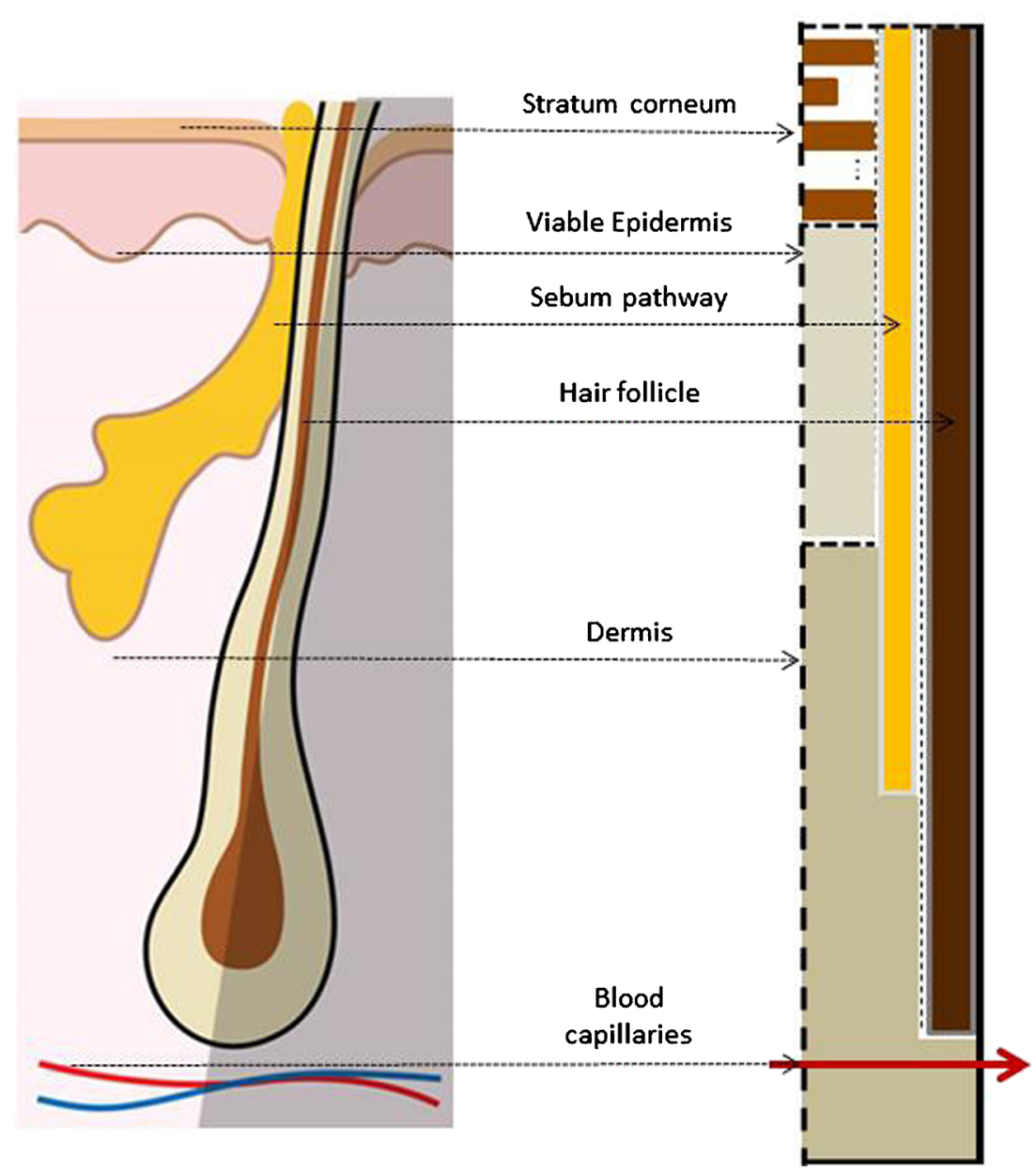

compartments considered in the computer model, where follicular penetration is simplified to be through the vertical sebum layer and the hair follicle itself is impermeable. In addition, the skin near a certain hair follicle is considered to be symmetric with respect to the hair follicle, and thus only half of the anatomy around hair (the unshaded area in Fig. 1a) needs to be modelled. The dimensions (and density with respect to skin surface area) of the hair follicle in this model are specific to body sites and can be set by the users, accounting for the variability from site to site on human body as reported in the literature $(39,40)$.

Figure $1 \mathrm{~b}$ also shows the other skin compartments around the hair follicle, which are needed for the integration of the follicular pathway with these compartments in modelling. The bricks-and-mortar structure of the stratum corneum can be seen at the top. Viable epidermis and dermis are modelled as homogeneous compartments; in the dermis compartment the systemic circulation is included as previously reported (19). Note that the sebaceous gland is not explicitly modelled. Further details regarding the integrated modelling framework are presented in the next section.

\section{The Integrated Mathematical Model}

Figure 2 shows the complete modelling framework where the hair follicle, shown on the right hand side of the schematic, is integrated with the rest of skin compartments. Briefly, on the top is a homogenous vehicle layer, followed by the bricks-andmortar structure of the stratum corneum (bricks: corneocytes; mortar: lipid), where the number of corneocyte layers, $\mathcal{N}$, is specific to body site. Further down are the homogeneous viable epidermis and dermis, and the blood capillaries in the dermis where the solute clearance into systemic circulation is calculated. With the full thickness skin, hair and blood capillary considered, this framework intends to model the transdermal permeation and kinetics in vivo. Simplifications compared to the real hair follicle arrangement have been made. The bending of the stratum corneum in the follicular orifices as well as the funnel-shaped infundibulum (39) were initially considered. Separate simulations showed that including such detailed representation of the hair follicle region had an impact in the local region but negligible effect on the overall transdermal permeation. Although in vivo studies concerning the role of follicular 
Fig. 2 Model framework for transdermal permeation and systemic bioavailability including the three pathways through the stratum corneum: intercellular (through lipid), transcellular, and follicular. Note that the hair itself, illustrated as the rightmost brown vertical area, is assumed impermeable. Not to scale.

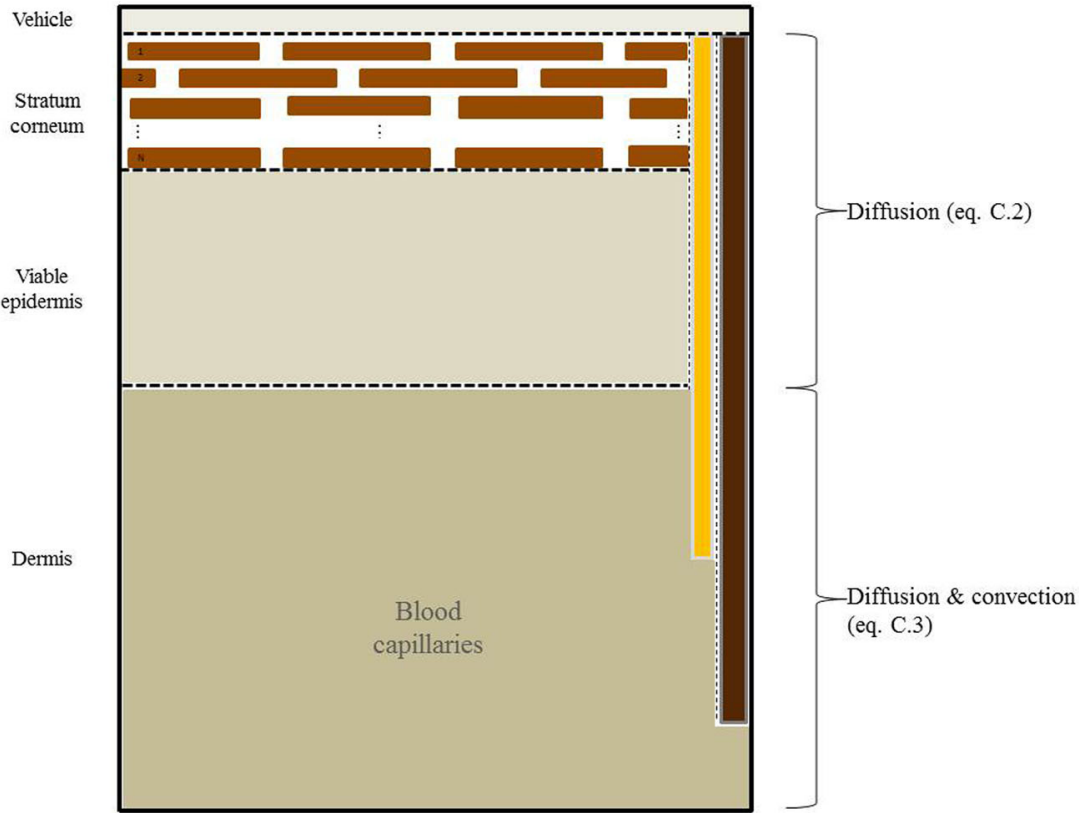

pathway are less common than in vitro ones, there is a general uncertainty regarding the suitability of in vitro tests for predicting in vivo situations with respect to the contribution of the follicular pathway to transdermal permeation mainly due to pre-processing of the samples $(41-43)$. Therefore, the focus of this study is to model the in vivo situations.

The governing equations include the diffusion equation that describes the transport of solute in all compartments as

Table I Model Input Parameters

\begin{tabular}{|c|c|c|}
\hline & Parameter & Value \\
\hline \multirow[t]{5}{*}{ Caffeine } & Molecular weight & 194.1906 Da \\
\hline & Octanol-water partition coefficient & 0.85 \\
\hline & Solubility in propylene glycol & $28.9 \mathrm{~kg} \mathrm{~m}^{-3}$ \\
\hline & Fraction of non-ionised solute & 0.99 \\
\hline & Fraction of unbound solute to albumin & 0.63 \\
\hline \multirow[t]{2}{*}{ Vehicle } & Thickness & $0.1912 \mu \mathrm{m}$ \\
\hline & Initial caffeine concentration & $37.35 \mathrm{~kg} \mathrm{~m}^{-3}$ \\
\hline \multirow[t]{6}{*}{ Stratum corneum } & Thickness & $14 \mu \mathrm{m}$ \\
\hline & Number of corneocyte layers & 16 (chest) \\
\hline & Width of corneocytes & $40 \mu \mathrm{m}$ \\
\hline & Height of corneocytes & $0.8 \mu \mathrm{m}$ \\
\hline & Thickness of intercellular lipid & $0.075 \mu \mathrm{m}$ \\
\hline & Lateral spacing between corneocytes & $0.075 \mu \mathrm{m}$ \\
\hline Viable epidermis & Thickness & $100 \mu \mathrm{m}$ \\
\hline Dermis & Thickness & $1000 \mu \mathrm{m}$ \\
\hline Hair follicle & Depth of HF & $610.57 \mu \mathrm{m}$ \\
\hline \multirow[t]{2}{*}{ Sebum } & Sebum tube width & $0.046 \mu \mathrm{m}^{*}$ \\
\hline & Sebum tube height & $410 \mu \mathrm{m}$ \\
\hline \multirow[t]{3}{*}{ Blood } & Cardiac output & $5.6 \mathrm{~L} \mathrm{~min}^{-1}$ \\
\hline & Skin blood flow as fraction of cardiac output & $5 \%$ \\
\hline & Caffeine clearance in blood & $0.078 \mathrm{Lh}^{-1} \mathrm{~kg}^{-1}$ \\
\hline \multirow[t]{2}{*}{ Subject } & Weight & $70 \mathrm{~kg}$ \\
\hline & Application area & $25 \mathrm{~cm}^{2}$ \\
\hline
\end{tabular}

\footnotetext{
*Value scaled down to match the reported ratio of surface area of hair follicle to that of skin; see text for details
} 


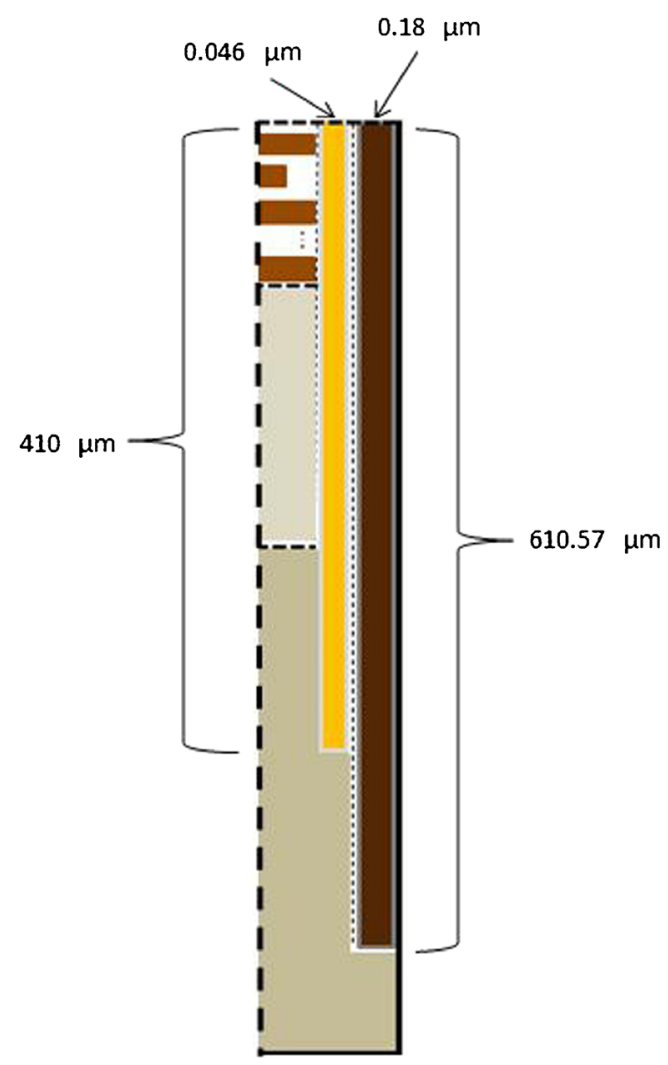

Fig. 3 Hair follicle region geometry presenting the width of hair and sebum layers. The numbers presented in the schematic are subjected to scaling. Detailed information is given in the text.

illustrated in Fig. 2. Inside dermis, mass transfer due to convection in blood is also included. Mass transfer calculation between the interfaces of compartments (e.g. between lipid and corneocyte in the stratum corneum, or between sebum and dermis) requires the partition coefficients of the solutes, as detailed in Appendix A and B. At the four boundaries of the entire modelling domain (solid lines in Fig. 2), zero flux is assumed. To solve these partial differential equations, in which the concentration of the solute changes with both spatial coordinates and time, the 2D domain in Fig. 2 is discretised into rectangular grids (19). Within each of the grids, the partial differential equation is converted to an ordinary differential equation (ODE) (Appendix $\mathrm{C}$ ) using the

Table II Diffusion and Partition Properties of Caffeine in Different Compartments

\begin{tabular}{lll}
\hline Skin compartment & $\begin{array}{l}\text { Diffusion coefficient } \\
\left(\mathrm{m}^{2} \mathrm{~s}^{-1}\right)\end{array}$ & $\begin{array}{l}\text { Partition coefficient with } \\
\text { respect to water }\end{array}$ \\
\hline Vehicle & $9.16 \times 10^{-10}$ & 0.87 \\
Lipid & $7.62 \times 10^{-12}$ & 0.80 \\
Corneocytes & $3.95 \times 10^{-15}$ & 2.56 \\
Sebum & $9.67 \times 10^{-11}$ & 0.06 \\
Viable epidermis \& Dermis & $1.85 \times 10^{-10}$ & 0.84 \\
\hline
\end{tabular}

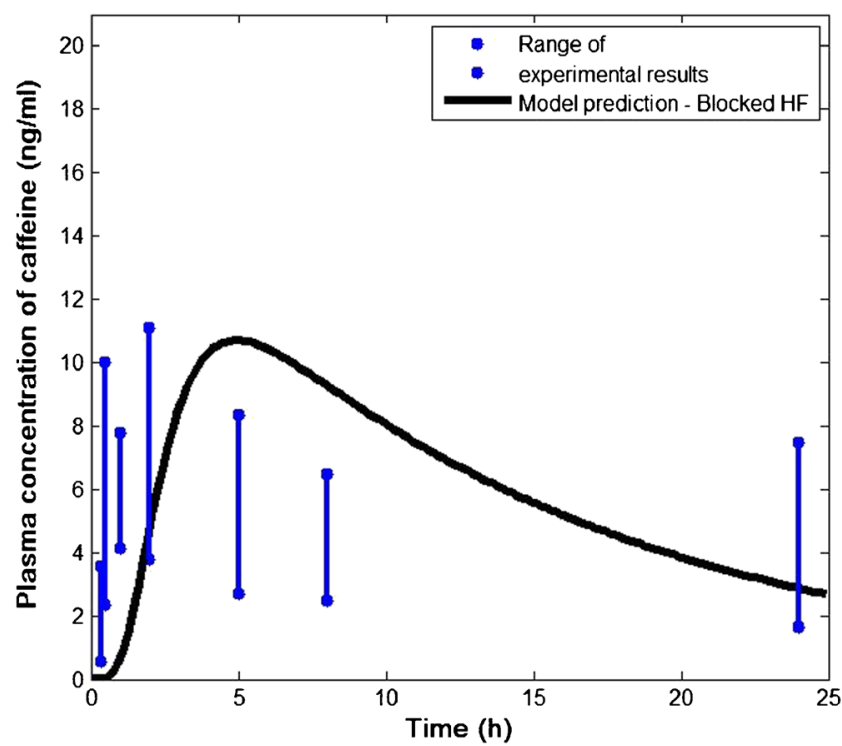

Fig. 4 Comparison of predicted plasma concentration of caffeine with published clinical data. In vivo data were obtained with blood samples taken from six human volunteers with blocked hair follicles (HF) (22).

standard method of lines (19). Due to constraint on space, detailed gridding is not presented, though it suffices to state that a large number of grids (1880 in total) are used to ensure that the results are independent of numerical inaccuracy due to this discretisation. The model was implemented in $\mathrm{C}++$ with ODEs solved by using the GVODE solver, a part of the SUNDIALS computational package (44).

The partition and diffusion coefficients in various compartments, except those for sebum, are obtained from the QSPR models calculated from the physicochemical properties of the solute and skin structures; these models have been published

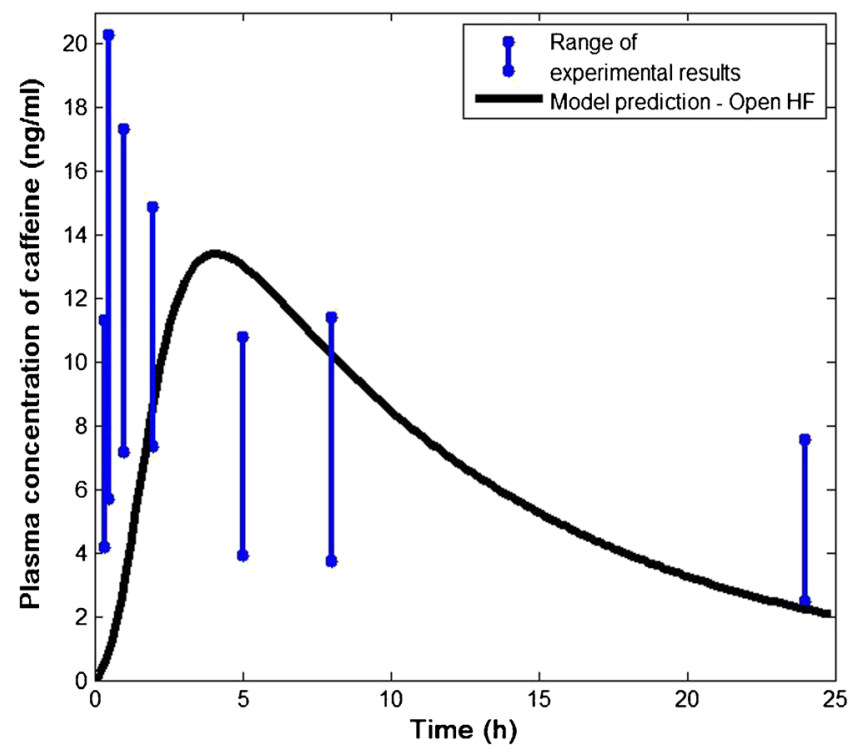

Fig. 5 Comparison of predicted plasma concentration of caffeine with published clinical data. In vivo data were obtained with blood samples taken from six human volunteers with open hair follicles (HF) (22). 


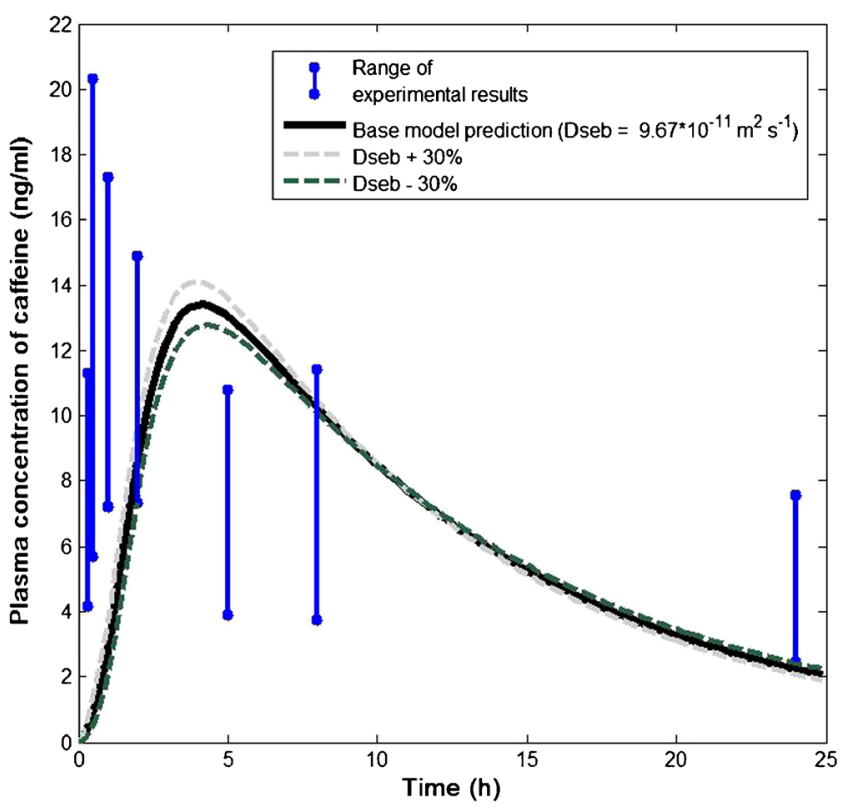

Fig. 6 Sensitivity analysis with respect to the diffusion coefficient of caffeine in sebum

in the literature and are collated in Appendix A and B. With the lack of reliable QSPR models for sebum, the experimentally measured partition and diffusion coefficients were used and will be discussed subsequently.

The vehicle of topical application is an important compartment that needs to be properly modelled, especially for in viwo finite-dose applications. In some cases (as in the caffeine case described below), the applied dose results in the concentration of the chemical in the vehicle exceeding its solubility in the corresponding solvent. The excess portion over solubility is modelled as solids. The rate of diffusion of the chemical from vehicle into skin is generally slower than the dissolution rate of solids in the vehicle. Therefore the vehicle is assumed to remain saturated until the excessive amount is fully dissolved (to make up the depletion of vehicle due to absorption into skin). The vehicle is then switched to a finite source in the model.

\section{Model Demonstration}

As demonstration, the model was applied to simulate the reported clinical study (22) of topically applied caffeine to human chest. In this experiment an ethanol and propylene glycol $(30: 70, v / v)$ solution containing $2.5 \%$ caffeine was applied to the chest of six healthy volunteers in two different set-ups: before and after blocking of the hair follicles with a wax-mixture. The application area was $25 \mathrm{~cm}^{2}$ with a dose of $2 \mathrm{mg}$ $\mathrm{cm}^{-2}$. The solution was left to evaporate, and since ethanol is known to evaporate within minutes, in the simulation the

Fig. 7 Predicted kinetics of transdermal delivery of caffeine with open and blocked hair follicles. (a) Caffeine permeated into the skin (b) caffeine disposited in the skin and (c) caffeine delivered to systemic circulation.

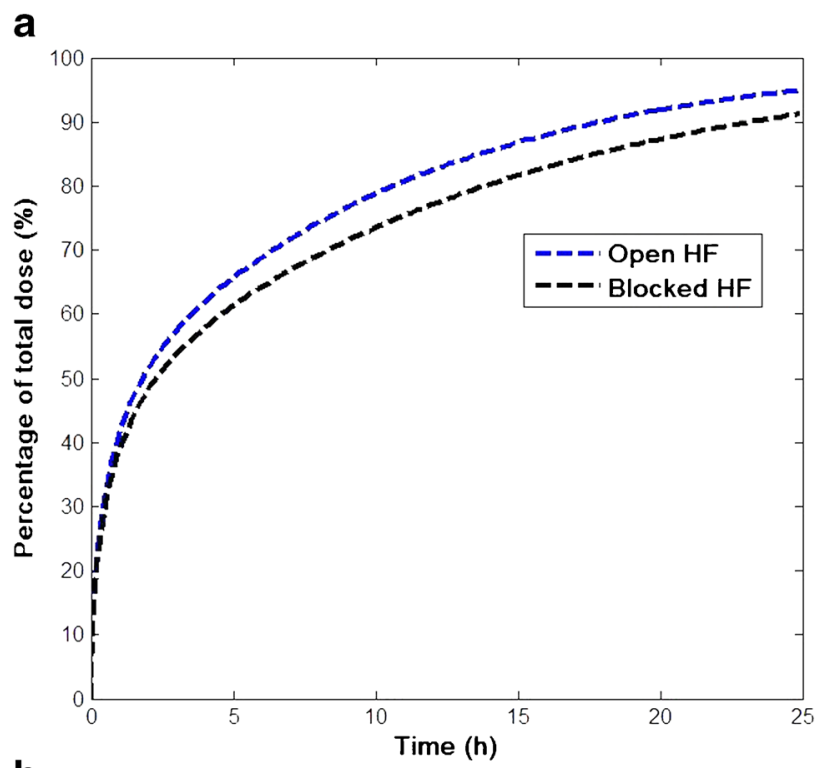

b

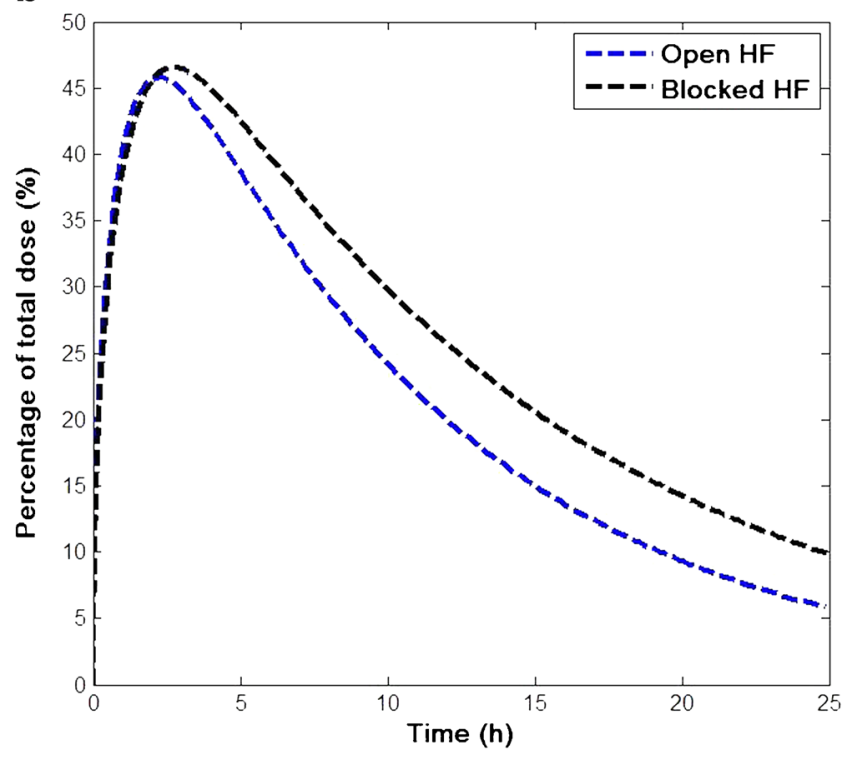

C

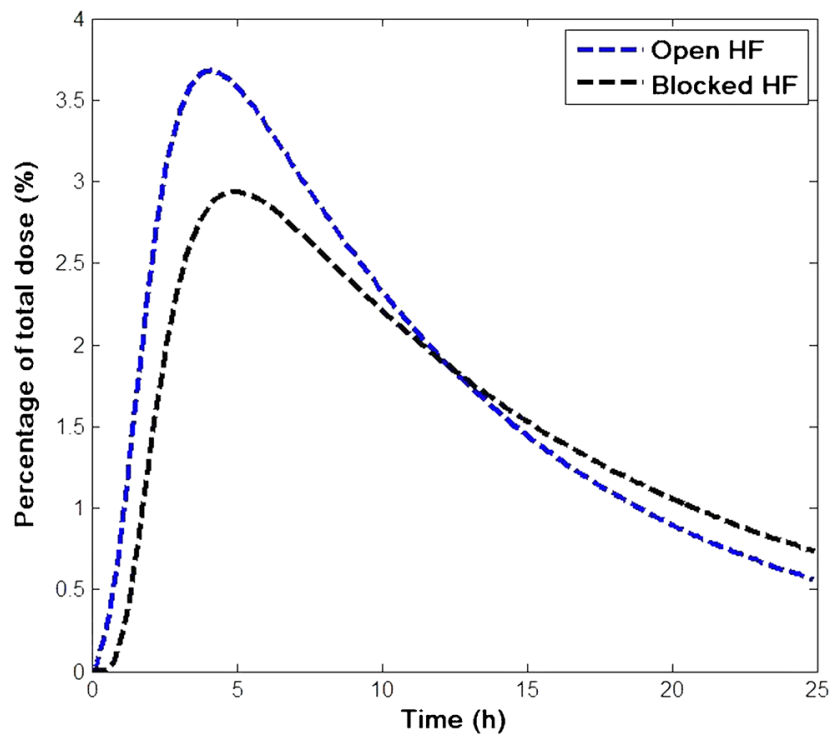


Table III Predicted Systemic Kinetics Following Topical Delivery of Caffeine with Open and Blocked Hair Follicles (HF). Percentage Change $=(O p e n$ HF - Blocked HF)/(Open HF)

\begin{tabular}{lllll}
\hline & $\begin{array}{l}C_{\max } \\
\left.(\mathrm{ng} \mathrm{mL})^{-1}\right)\end{array}$ & $\begin{array}{l}\mathrm{T}_{\max } \\
(\mathrm{h})\end{array}$ & $\begin{array}{l}\mathrm{AUC}(\mathrm{I} \mathrm{h}) \\
\left.(\mathrm{ng} \mathrm{h} \mathrm{mL})^{-1}\right)\end{array}$ & $\begin{array}{l}\mathrm{AUC}(\mathrm{IO} \mathrm{h}) \\
\left.(\mathrm{ng} \mathrm{h} \mathrm{mL})^{-1}\right)\end{array}$ \\
\hline Open HF & 13.40 & 4.0 & 0.86 & 97 \\
Blocked HF & 10.7 & 5.0 & 0.10 & 77 \\
Percentage change & $20 \%$ & $-25 \%$ & $88 \%$ & $21 \%$ \\
\hline
\end{tabular}

vehicle was simplified to consist of only propylene glycol and caffeine. During the experiments, blood samples were taken after each application at different times. The measured concentration of caffeine in the plasma with and without follicular blocking was reported to show the contribution of the follicular pathway to the overall transdermal penetration. This plasma concentration profile will be compared with model predictions.

The input parameters used for simulation are listed in Table I, where the physicochemical properties listed are used to calculate the partition and diffusion coefficients as detailed in Appendix A and B. The dimensions of skin including the hair follicle are chest specific. Specifically, the stratum corneum thickness in chest was set to $14 \mu \mathrm{m}$ (45). The viable epidermis thickness is set to $100 \mu \mathrm{m}$ and dermis thickness $1000 \mu \mathrm{m}$ (46). Regarding the follicular pathway, the vertical depth of the hair is set to be $610.57 \mu \mathrm{m}(20)$. The vertical depth of the sebum is set to be $410 \mu \mathrm{m}$ which is approximately the depth of the sebaceous glands in the skin (47). The radius of hair in the thorax was reported to be ca. $40 \mu \mathrm{m}(48)$ and that of the hair follicle opening $50 \mu \mathrm{m}$ (49). This suggests that the lateral width of the sebum layer in Fig. $1 \mathrm{~b}$ is $10 \mu \mathrm{m}$, by assuming that sebum completely fills the space between hair and the follicular opening. Furthermore, Otberg et al. (49) showed that the average area of follicular orifices in thorax is $0.19 \%$ of the skin surface. To directly represent this ratio using the actual diameter of hair follicle with a computer model would require a large simulation domain and computational power. Here, the width of the stratum corneum (and the viable epidermis and dermis beneath) is kept to three corneocytes width (i.e. $120 \mu \mathrm{m}$ ) to save computation expenses. Accordingly the width of sebum is scaled down to $0.046 \mu \mathrm{m}$ to meet the above ratio. (Detailed calculation is as follows. Given the lateral width of stratum corneum of $120 \mu \mathrm{m}$ and the surface area ratio of $0.19 \%$, the entire follicle opening radius is $0.19 \% \times 120 /(1-0.19 \%)=0.228 \mu \mathrm{m}$. Furthermore, it was found that the radius of hair in chest is ca. $40 \mu \mathrm{m}$ (48) and that of the hair follicle opening $50 \mu \mathrm{m}$ (49), suggesting the sebum annulus radius is $1 / 5$ of the entire follicle opening. Therefore in the scaled geometry the sebum annulus width is determined to be $0.228 / 5=0.046 \mu \mathrm{m}$.) The dimensions of the hair follicle are summarised in Fig. 3.

The partition and diffusion coefficients of caffeine in various skin compartments (except in sebum) were obtained from the established QSPR equations detailed in Appendix A and $\mathrm{B}$, using the physicochemical properties of caffeine given in Table I. The partition and diffusion coefficients in sebum were reported for some chemicals but the QSPR models developed had substantial uncertainty in the prediction $(35,36)$. In the present study, the diffusion coefficient was set to be the same as the measured data of butyl 4-hydroxybenzonate $\left(9.67 \times 10^{-11} \mathrm{~m}^{2} \mathrm{~s}^{-1},(36)\right)$, which has the same molecular weight as caffeine; according to Mitragotri (50) the diffusion coefficient in a certain media is primarily determined by the molecular weight of the chemical. The sebum:water partition coefficient of caffeine was obtained from a standard equilibrium experiment conducted at the China Agricultural University (private communications). The clearance rate of caffeine in systemic circulation was based on the reported data for oral delivery $0.078 \mathrm{~L} \mathrm{~h}^{-1} \mathrm{~kg}^{-1}$ (51). The vehicle:water partition coefficient is estimated to be 0.87 from the solubility of caffeine in the vehicle over that in water (16). The diffusion coefficient of caffeine in the vehicle was estimated to be $9.16 \times 10^{-10} \mathrm{~m}^{2} \mathrm{~s}^{-1}$ using the Stokes-Einstein equation (Eq. B.1). Table II summarises the diffusion and partition properties of caffeine in different compartments.

\section{RESULTS AND DISCUSSION}

The predicted plasma concentration of caffeine is shown in Fig. 4 (blocked hair follicle) and Fig. 5 (open hair follicle) in comparison with the published experimental data (22). The range of the concentrations was obtained from six subjects reflecting significant inter-subject variability. In both cases, the model prediction appears to be in good agreement with the in vivo data.

Subsequently, sensitivity analysis was conducted to explore the impact of the parameter variability relating to sebum on model predictions. Sensitivity analysis respect to other skin compartments has been reported elsewhere (e.g. (19)). Figure 6 shows the predicted systemic kinetics when the diffusion coefficient of caffeine in sebum is subjected to $30 \%$ variability. Clearly, decreasing (increasing) the diffusion coefficient results in slower (faster) penetration of caffeine into the blood, since the penetration through the follicular pathway becomes slower (faster). A similar effect was observed when subjecting the partition coefficient and sebum width to similar extent of variability (results not reported).

Figure 7 illustrates the predicted kinetics of caffeine absorbed from the vehicle (Fig. 7a), disposition in skin (Fig. $7 \mathrm{~b}$, all skin compartments except blood), and delivery into systemic circulation (Fig. 7c), in terms of the percentage of total dose applied for open and blocked hair follicles. Caffeine is mildly hydrophilic and thus its partitioning into oily sebum is substantially less than partitioning into either lipid or corneocytes in stratum corneum (c.f. Table II). 

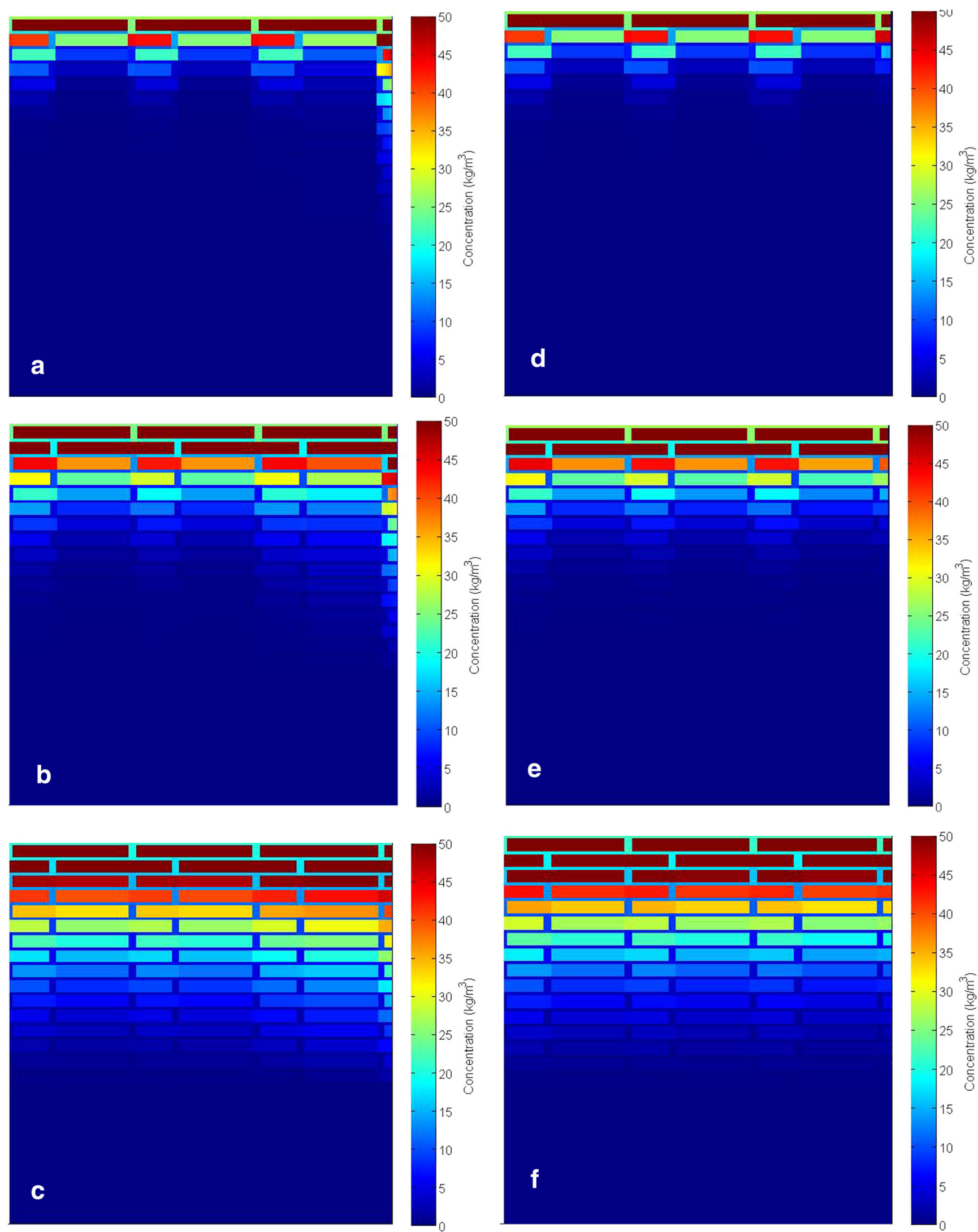

Fig. 8 Subcellular disposition of caffeine in the stratum corneum of the skin with open $(\mathbf{a}, \mathbf{b}, \mathbf{c})$ and blocked $(\mathbf{d}, \mathbf{e}, \mathbf{f})$ hair follicles (HF) at $5 \mathrm{~min}(\mathbf{a}, \mathbf{d}), 20 \mathrm{~min}(\mathbf{b}, \mathbf{e})$ and I h (c, f) after application. 


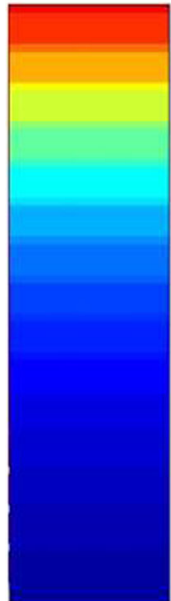

a

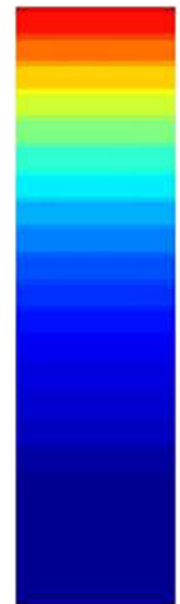

b

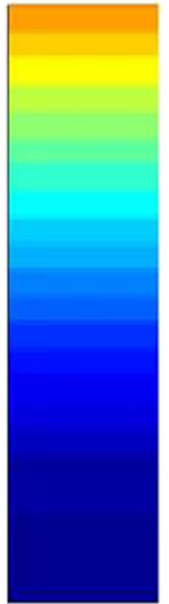

d

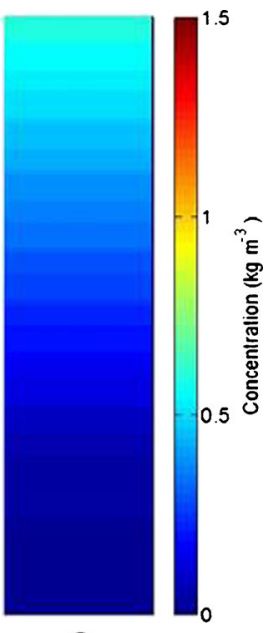

e
Fig. 9 Disposition of caffeine in the sebum layer when hair follicles are open; (a) $t=5 \mathrm{~min} ;(\mathbf{b}) t=20 \mathrm{~min} ;(\mathbf{c}) t=1 \mathrm{~h} ;(\mathbf{d}) t=10 \mathrm{~h}$.

However, the diffusion coefficient in sebum is $10+$ times higher than that in lipid (and several orders of magnitude higher than in corneocytes). As a result, the overall effect of the follicular pathway is significant. It can be seen from Fig. 7a that when hair follicles are open, a greater and faster uptake of caffeine by the skin is observed. Figure $7 \mathrm{~b}$ shows that, due to the additional follicular pathway that bypasses the stratum corneum to reach viable epidermis and dermis, caffeine resides for less time in the skin with faster and higher delivery to the blood (Fig. 7c), when compared with the situation where hair follicles are blocked.

In Table III the relative contribution of the follicular pathway to caffeine delivery to systemic circulation, as predicted by the model, is quantified in terms of the maximum plasma concentration $\left(\mathrm{C}_{\max }\right)$, the time to reach $\mathrm{C}_{\max }\left(\mathrm{T}_{\max }\right)$ and the area under curve (AUC). AUC represents the overall systemic bioavailability of dermal exposure for a given time. These values clearly show that hair follicles contribute significantly to the overall transdermal permeation especially at the early stage of application. Specifically, the AUC in systemic circulation one hour after application is substantially higher when hair follicles are open $\left(0.86 \mathrm{ng} \mathrm{h} \mathrm{mL}^{-1}\right)$ then when hair follicles are blocked $\left(0.1 \mathrm{ng} \mathrm{h} \mathrm{mL}{ }^{-1}\right)$, with a percentage difference of $88 \%$. Even at $10 \mathrm{~h}$ post application, the percentage difference of AUC between blocked and open hair follicles is still very significant, at $21 \%$. The difference in $\mathrm{C}_{\max }$ and $\mathrm{T}_{\max }$ is also significant. It is worth noting that the predicted difference also agreed to a large extent with the clinical study (22). These results highlight the importance of hair follicles for the bioavailability in the skin and systemic circulation after dermal exposure to caffeine.

Figure 8 presents the detailed 2D disposition of caffeine in the stratum corneum predicted by the model at different time steps after the application of caffeine. The two sections of the figure represent the simulations with the follicular pathway open (a-c) and blocked (d-e). The concentration profiles clearly show the contribution of the follicular pathway in the permeation process. Caffeine concentration in the corneocytes is visibly higher than in the lipid and this is due to the high level of binding of caffeine in corneocytes compared to any other compartment (Table II). At early times (e.g. up to $20 \mathrm{~min}$ ), the concentration profiles are noticeably different with the follicular route being of significant importance in penetration.

Due to the relatively low partition coefficient in sebum, the disposition of caffeine in sebum is not apparent in Fig. 8a-c. In Fig. 9, the concentration profile in sebum is rescaled and shown for different times. As can be seen from this figure, high concentrations are observed during the early stage after application whereas as time proceeds, caffeine concentration in sebum decreases. This, together with the overall penetration profile illustrated in Figs. 5, 6, and 7, suggests that the impact of follicular pathway on caffeine delivery is more significant at the early times after application. This observation from model prediction agrees with experimental studies in the literature, e.g. $(21,22)$

\section{CONCLUSIONS}

This paper presents a new in silico model for transdermal permeation and systemic bioavailability with the integration of the follicular pathway. The multi-scale model considers the important molecular and microscopic principles involved in skin permeation and systemic absorption. To our knowledge, this is the first model in the open literature that has the capability to offer quantitative prediction of the three major pathways (intercellular, intracellular and follicular) of transdermal permeation. The model confirms the importance of the follicular pathway. Prediction of the disposition of chemicals in various skin compartments enhances our understanding of the local pharmaco-/toxico-kinetics after skin exposure for assessing efficacy and toxicity. This model could provide improved in silico screening for pharmaceuticals and industrial chemicals and be a valuable tool in extrapolating from in vitro experiments to in vivo exposure conditions - a key component to reduce the reliance on animal models. It should be noted that the model is applicable to topical application of small molecules $(<500 \mathrm{Da})$ on a given body site, provided that the appropriate physiological parameters, physicochemical properties of the solute and vehicle properties are specified. Further validation of the model with experimental studies of more compounds and/or exposure scenarios is needed. Currently, a more comprehensive validation of the developed model against advanced imaging data is being planned.

\section{ACKNOWLEDGMENTS AND DISCLOSURES}

This work was supported by the UK Biotechnology and Biological Sciences Research Council (grant number: BB/ L502042/1), and Unilever Research Colworth, UK. We 
would like to thank Dr. Scott Singleton at the Strategic Science Group, Unilever, for his valuable insight to this project, and Professor Lujia Han's group at the China Agricultural University for sharing unpublished sebum partition data.

\section{APPENDIX A: PHYSICAL PROPERTIES}

These properties are required in the QSPR models in Appendix B to predict partition and diffusion coefficients in various compartments of skin, and mass transfer into blood.

\section{A.I. Solute radius}

The radius of the solute $(\AA)$ is estimated using the equation below based on the molecular weight (MW) of the solute (52):

$r_{\mathrm{s}}=\sqrt[3]{3 / 4 \pi \times 0.9087 \mathrm{MW}}$

\section{A.2. Volume fraction of water in stratum corneum at saturation}

Swelling and shrinking of the stratum corneum (SC) is not considered in this study (14) thus the porosities of both SC lipids and corneocytes are considered to be constant. Here the porosity is defined as the volume fraction of the SC pores at fully dehydrated state. The volume fraction of water in the $\mathrm{SC}$ at saturation $\left(\varphi_{\mathrm{sc}}\right)$ is related to the volume of lipid $\left(\mathrm{V}_{1}\right)$, keratin $\left(\mathrm{V}_{\mathrm{k}}\right)$, and saturated water $\left(\mathrm{V}_{\mathrm{w}}\right)$, content by $\varphi_{\mathrm{sc}}=\mathrm{V}_{\mathrm{w}} /$ $\left(\mathrm{V}_{\mathrm{w}}+\mathrm{V}_{\mathrm{l}}+\mathrm{V}_{\mathrm{k}}\right)$. The water phase distributed into the SC lipids and corneocytes $\left(\mathrm{V}_{\mathrm{w}}=\mathrm{V}_{\mathrm{wl}}+\mathrm{V}_{\mathrm{wk}}\right)$ with the corresponding saturated volume fraction of water (or porosity) in SC lipids and corneocytes as $\varphi_{\mathrm{m}}=\mathrm{V}_{\mathrm{wl}} /\left(\mathrm{V}_{\mathrm{wl}}+\mathrm{V}_{\mathrm{l}}\right)$ and $\varphi_{\mathrm{b}}=\mathrm{V}_{\mathrm{wk}} /\left(\mathrm{V}_{\mathrm{wk}}+\mathrm{V}_{\mathrm{k}}\right)$. Since $\mathrm{V}_{\mathrm{w}}+\mathrm{V}_{\mathrm{l}}+\mathrm{V}_{\mathrm{k}}=\mathrm{V}_{\mathrm{wl}}+\mathrm{V}_{\mathrm{l}}+\mathrm{V}_{\mathrm{wk}}+\mathrm{V}_{\mathrm{k}}$, it follows that $\mathrm{V}_{\mathrm{w}}$ / $\varphi_{\mathrm{sc}}=\mathrm{V}_{\mathrm{wl}} / \varphi_{\mathrm{m}}+\mathrm{V}_{\mathrm{wk}} / \varphi_{\mathrm{b}}$. Using the relationship $\mathrm{V}=\mathrm{m} / \rho$ (where $\mathrm{m}$ is the mass and $\rho$ is the density), it follows that porosity of the $\mathrm{SC}$ is related to the porosities of SC lipids and corneocytes by (14):

$\frac{\mathrm{f}_{\mathrm{l}} / \rho_{\mathrm{l}}+\mathrm{f}_{\mathrm{k}} / \rho_{\mathrm{k}}}{\mathrm{I}-\boldsymbol{\phi}_{\mathrm{sc}}}=\frac{\mathrm{f}_{\mathrm{l}} / \rho_{\mathrm{l}}}{\mathrm{I}-\boldsymbol{\phi}_{\mathrm{m}}}+\frac{\mathrm{f}_{\mathrm{k}} / \rho_{\mathrm{k}}}{\mathrm{I}-\boldsymbol{\phi}_{\mathrm{b}}}$

where $\mathrm{f}_{\mathrm{l}}(\approx 12.5 \%)$ and $\mathrm{f}_{\mathrm{k}}(\approx 87.5 \%)$ are the dry mass fractions of $\mathrm{SC}$ lipid and keratin. The overall porosity of the SG can be related to its saturated water content (mass fraction) by the following equation:

$\phi_{\mathrm{sc}}=\frac{\mathrm{f}_{\mathrm{sc}}}{\rho_{\mathrm{w}}\left(l-\mathrm{f}_{\mathrm{sc}}\right)\left(\mathrm{f}_{\mathrm{l}} / \rho_{\mathrm{l}}+\mathrm{f}_{\mathrm{k}} / \rho_{\mathrm{k}}\right)+\mathrm{f}_{\mathrm{sc}}}$

In order to estimate the above parameters, the user needs to enter the thickness of the SC. The dimensions and offset of bricks and mortars in the SC are fixed according to our previous study (14).

\section{A.3. The hydraulic permeability of the medium (k)}

The hydraulic permeability is estimated from the correlation derived from Jackson and James (53) given as:

$\mathrm{k}=\beta \mathrm{r}_{\mathrm{f}}^{2}\left(\mathrm{I}-\theta_{\mathrm{b}}\right)^{\gamma}$

where $\beta$ and $\gamma$ are fitting parameters that will be explained later on and $\theta_{\mathrm{b}}$ is the actual fraction of water in the corneocyte phase. $r_{\mathrm{f}}$ is the radius ok keratin microfibril $\left(\mathrm{r}_{\mathrm{f}}=3.5 \mathrm{~nm}(54)\right)$

\section{A.4 Volumetric blood flow}

The volumetric blood flow $(\mathrm{Q}$ can be estimated according to physiology. It is known that the average resting cardiac output is ca. 5.6 $\mathrm{L} \mathrm{min}^{-1}$ for a human male and $4.9 \mathrm{~L} \mathrm{~min}^{-1}$ for a female (55). The overall blood flow to skin is estimated to be $5 \%$ of the cardiac output (56). Therefore for a male and female respectively the equations used are shown below:

\section{APPENDIX B: DIFFUSION AND PARTITION PROPERTIES}

\section{B.I Vehicle properties}

Diffusion coefficient in vehicle and any other medium can be calculated using the Stokes-Einstein equation (57):

$\mathrm{D}_{\mathrm{w}}=\frac{\mathrm{KT}}{6 \pi \eta \mathrm{r}_{\mathrm{s}}}$

where $\mathrm{K}$ is the Boltzmann constant, $\mathrm{T}$ is the room temperature $(309 \mathrm{~K}), \eta$ is the viscosity of the medium and $r_{\mathrm{s}}$ is the solute radius as calculated in Eq. (A.1).

Partition coefficient in the vehicle is calculated using the solubility of the chemical in water and the solubility of the chemical in the vehicle. If the vehicle is water then it equals 1 .

\section{B.2. Lipid properties in stratum corneum}

Diffusion coefficient in the SC lipid is related to the solute radius (A.1) by the equation proposed by Mitragotri (50):

$$
\mathrm{D}_{\mathrm{m}}= \begin{cases}2 \times 10^{-9} \exp \left(-0.46 \mathrm{r}_{\mathrm{s}}^{2}\right), & \mathrm{MW} \leq 380 \mathrm{Da} \\ 3 \times 10^{-13}, & \mathrm{MW}>380 \mathrm{Da}\end{cases}
$$


The partition coefficient of lipid to water can be estimated using the equation below (17):

$\mathrm{K}_{\mathrm{mw}}=\frac{\rho_{\mathrm{l}}}{\rho_{\mathrm{w}}} \mathrm{K}_{\mathrm{ow}}^{0.69}$

Where $\rho_{\mathrm{l}}=0.9 \mathrm{~g} / \mathrm{cm}^{3}$.

\section{B.3. Corneocyte properties in stratum corneum}

Solute diffusion coefficient in the $\mathrm{SC}$ corneocytes $\left(\mathrm{D}_{\mathrm{b}}\right)$ is estimated in the same way as our previous study (14):

$$
\mathrm{D}_{\mathrm{b}}=\frac{\exp \left(-\alpha \mathrm{S}^{\lambda}\right)}{1+\frac{\mathrm{r}_{\mathrm{s}}}{\sqrt{\mathrm{k}}}+\frac{\mathrm{r}_{\mathrm{s}}^{2}}{3 \mathrm{k}}} \times \mathrm{D}_{\mathrm{w}}
$$

where $\alpha, \beta, \gamma$ and $\lambda$ are model fitting parameters $(\alpha=9.47$, $\beta=9.32 \times 10^{-8}, \gamma=-1,17$ and $\left.\lambda=1,09\right), S=\left(1-\theta_{b}\right)\left(\frac{r_{s}+r_{f}}{r_{f}}\right)^{2}$, $\mathrm{k}$ is the hydraulic permeability (Eq. (A.3)) and $\mathrm{D}_{\mathrm{w}}$ the diffusion coefficient in water (Eq. (B.2)). The fitting parameters $\alpha, \beta, \gamma$ and $\lambda$ were chosen after applying the above QSPR model to fit steady-state skin permeability data in the respective studies cited above.

The partition coefficient of corneocyte to water is estimated using the following equation (14):

$\mathrm{K}_{\mathrm{bw}}=\left(\mid-\phi_{\mathrm{b}}\right) \mathrm{K}_{\mathrm{kw}}+\theta_{\mathrm{b}}$

where $\mathrm{K}_{\mathrm{kw}}$ is the solute binding constant to SC keratin and is estimated using the following reported eq. (58):

$\mathrm{K}_{\mathrm{kw}}=\frac{\rho_{\mathrm{k}}}{\rho_{\mathrm{w}}} \times 4.2 \mathrm{~K}_{\mathrm{ow}}^{0.31}$

where $\rho_{\mathrm{k}}=1.37 \mathrm{~g} / \mathrm{cm}^{3}$ and $\rho_{\mathrm{w}}=1 \mathrm{~g} / \mathrm{cm}^{3}$. In order to get this coefficient the user is required to enter the Octanol water partition coefficient of the chemical to be tested.

\section{B.4. Viable epidermis and dermis properties}

As literature suggests that viable epidermis and dermis have similar multiphase compositions (16,59) the partition and diffusion properties in these two layers are assumed to be similar. The partition coefficient is calculated from the following equation proposed by Kretsos et al. in 2008 (59) and then modified by Ibrahim et al. in 2012 (60).

$\mathrm{K}_{\mathrm{v}}=0.7 \times\left(0.68+\frac{0.32}{\mathrm{f}_{\mathrm{u}}}+0.025 \mathrm{f}_{\text {non }} \mathrm{K}_{\mathrm{ow}}{ }^{0.7}\right)$

where the three terms $0.68,0.32 / f_{u}$ and $0.025 f_{\text {non }}$ account for the chemical disposition in albumin accessible aqueous phase. $f_{\text {non }}$ is the fraction of solute non-ionized in the aqueous phase and $f_{u}$ is the fraction of unbound (to albumin) solute.

The diffusion coefficient is also based on the binding/ partitioning properties of the dermis and is given from the equation below:

$\mathrm{D}_{\mathrm{v}}=\frac{10^{-8.15-0.655 \log \mathrm{MW}}}{0.68+\frac{0.32}{\mathrm{f}_{\mathrm{u}}}+0.025 \mathrm{f}_{\mathrm{non}} \mathrm{K}_{\mathrm{mw}}}$

\section{APPENDIX C: OVERALL SIMULATION APPROACH}

The mass transfer equation is solved by a finite difference scheme. In order to do so the skin is divided into a finite number of smaller elements.

The mass transferred between neighbouring elements A and $\mathrm{B}$ is given by (14):

$$
\mathrm{q}_{\mathrm{AB}}=\frac{\mathrm{A}_{\mathrm{i}}}{\frac{\delta_{\mathrm{A}}}{\mathrm{D}_{\mathrm{A}}}+\frac{\mathrm{K}_{\mathrm{AB}} \delta_{\mathrm{B}}}{\mathrm{D}_{\mathrm{B}}}}\left(\mathrm{C}_{\mathrm{A}}-\mathrm{K}_{\mathrm{AB}} \mathrm{C}_{\mathrm{B}}\right)
$$

where $\mathrm{q}_{\mathrm{AB}}$ is the flux of solute from grid $\mathrm{A}$ to grid $\mathrm{B}, \mathrm{A}_{\mathrm{i}}$ is the interfacial area between the two grids, $\delta_{\mathrm{A}}$ and $\delta_{\mathrm{B}}$ are the corresponding diffusion length, $\mathrm{D}_{\mathrm{A}}$ and $\mathrm{D}_{\mathrm{B}}$ are the diffusion coefficients of the two elements, $\mathrm{K}_{\mathrm{AB}}$ is the solute partition coefficient between the two elements $\left(\mathrm{K}_{\mathrm{AB}}=1\right.$ if the two grids are the same element and $\mathrm{K}_{\mathrm{AB}}=\mathrm{K}_{\mathrm{Aw}} / \mathrm{K}_{\mathrm{Bw}}$ if element $\mathrm{A}$ is different from $\mathrm{B}), \mathrm{C}_{\mathrm{A}}$ and $\mathrm{C}_{\mathrm{B}}$ are the solute concentrations in the two elements.

According to mass conservation principles, concentration of each element A satisfies the following equation:

$\frac{\mathrm{dC}_{\mathrm{A}}}{\mathrm{dt}_{\mathrm{s}}}=-\frac{\sum \mathrm{q}_{\mathrm{AB}}}{\mathrm{V}}$

where $\mathrm{C}_{\mathrm{A}}$, is the solute concentration in element $\mathrm{A}, \mathrm{V}$ is the element volume, $\mathrm{t}_{\mathrm{S}}$ is the time, $\mathrm{q}_{\mathrm{AB}}$ is the flux of solute into/out of element A from neighbouring element B.

When it comes to dermis in addition to diffusion, convection needs to be considered when modelling the transport between dermis and the capillaries due to blood flow. The full explanation of this approach can be found in the respective reference (19). For a dermis grid I, the mass balance equation is:

$\mathrm{V}_{\mathrm{A}} \frac{\mathrm{dC}_{\mathrm{A}}}{\mathrm{dt}}=-\sum \mathrm{q}_{\mathrm{AB}}+\mathrm{Q}_{\mathrm{b}, \mathrm{A}}\left(\mathrm{C}_{\mathrm{b}}-\frac{\mathrm{C}_{\mathrm{A}}}{\mathrm{K}_{\mathrm{db}}}\right)$

where $Q_{b}, A$ is the volumetric blood flow into dermis grid $A, C_{b}$ is the solute concentration in the blood and $\mathrm{K}_{\mathrm{db}}$ is the partition coefficient from dermis to blood. 
The systemic circulation and clearance is described by the following equation:

$\mathrm{V}_{\mathrm{b}} \frac{\mathrm{dG}_{\mathrm{b}}}{\mathrm{dt}}=\mathrm{N} * \sum\left[\mathrm{Q}_{\mathrm{b}, \mathrm{A}} *\left(\frac{\mathrm{C}_{\mathrm{A}}}{\mathrm{K}_{\mathrm{db}}}-\mathrm{C}_{\mathrm{b}}\right)\right]-\mathrm{KG}_{\mathrm{b}}$

where $V_{b}$ is the volume of the whole body blood vessel, and $\mathrm{KC}_{\mathrm{b}}$ is the first order clearance that may include transport into other tissues and metabolism.

Open Access This article is distributed under the terms of the Creative Commons Attribution 4.0 International License (http://creativecommons.org/licenses/by/4.0/), which permits unrestricted use, distribution, and reproduction in any medium, provided you give appropriate credit to the original author(s) and the source, provide a link to the Creative Commons license, and indicate if changes were made.

\section{REFERENCES}

1. Godin B, Touitou E. Transdermal skin delivery: predictions for humans from in vivo, ex vivo and animal models. Adv Drug Deliv Rev. 2007;59(11):1152-61.

2. Zhong W, Xing MMQ Hui X, Maibach HI. A stochastic model for transepidermal drug delivery. Skin Res Technol. 2009;15(4): 407-11.

3. European Commission. Regulation (EC) 1223/2009 of the European Parliament and of the council of 30 November 2009 on cosmetic products. Off J Eur Communities. 2009;L342:59. 22 Dec 2009

4. Frasch HF, Barbero AM. Application of numerical methods for diffusion-based modeling of skin permeation. Adv Drug Deliv Rev [Internet] Elsevier BV. 2013;65(2):208-20.

5. Potts RO, Guy RH. Predicting skin permeability. Pharm Res An Off J Am Assoc Pharm Sci. 1992;9(5):663-9.

6. Anissimov YG, Jepps OG, Dancik Y, Roberts MS. Mathematical and pharmacokinetic modelling of epidermal and dermal transport processes. Adv Drug Deliv Rev Elsevier BV. 2013;65(2):169-90.

7. Parry GE, Bunge AL, Silcox GD, Pershing LK, Pershing DW. Percutaneous absorption of benzoic-acid across human skin.1. In vitro experiments and mathematical-modeling. Pharm Res. 1990;7(3):230-6.

8. Guy RH, Hadgraft J. A theoretical description relating skin penetration to the thickness of the applied medicament. Int J Pharm. 1980;6(3-4):321-32.

9. Krüse J, Golden D, Wilkinson S, Williams F, Sanja Kezic JC. Analysis, interpretation, and extrapolation of dermal permeation data using diffusion-based mathematical models. J Pharm Sci. 2007;96(3):682-703.

10. Kasting G. Kinetics of finite dose absorption through skin 1 . Vanillylnonanamide J Pharm Sci. 2001;90(2):202-12.

11. Rim JE, Pinsky PM, van Osdol WW. Using the method of homogenization to calculate the effective diffusivity of the stratum corneum with permeable corneocytes. J Biomech. 2008;41(4): 788-96.

12. Hansen S, Henning A, Naegel A, Heisig M, Wittum G, Neumann $\mathrm{D}$, et al. In-silico model of skin penetration based on experimentally determined input parameters. Part I: experimental determination of partition and diffusion coefficients. Eur J Pharm Biopharm. 2008;68(2):352-67.

13. Wang T, Kasting G, Nitsche J. A multiphase microscopic diffusion model for stratum corneum permeability. I. Formulation, solution, and illustrative results for representative compounds. J Pharm. 2006;95(3):620-48.

14. Chen L, Lian G, Han L. Use of "bricks and mortar" model to predict transdermal permeation: model development and initial validation. Ind Eng Chem Res. 2008;47(17):6465-72.

15. Chen L, Lian G, Han L. Modeling transdermal permeation. Part I. Predicting Skin Permeability of Both Hydrophobic and Hydrophilic Solutes. 2010;56(5):1136-46.

16. Dancik Y, Miller MA, Jaworska J, Kasting GB. Design and performance of a spreadsheet-based model for estimating bioavailability of chemicals from dermal exposure. Adv Drug Deliv Rev Elsevier BV. 2013;65(2):221-36.

17. Chen L, Han L, Saib O, Lian G. In silico prediction of percutaneous absorption and disposition kinetics of chemicals. Pharm Res. 2015;32(5):1779-93.

18. Jepps OG, Dancik Y, Anissimov YG, Roberts MS. Modeling the human skin barrier-towards a better understanding of dermal absorption. Adv Drug Deliv Rev Elsevier BV. 2013;65(2):152-68.

19. Chen T, Lian G, Kattou P. In silico modelling of transdermal and systemic kinetics of topically applied solutes: model development and initial validation for transdermal nicotine. Pharm Res Pharmaceutical Research. 2016;33(7):1602-14.

20. Knorr F, Lademann J, Patzelt A, Sterry W, Blume-Peytavi U, Vogt A. Follicular transport route-research progress and future perspectives. Eur J Pharm Biopharm Elsevier BV. 2009;7 1(2):173-80.

21. Liu X, Grice JE, Lademann J, Otberg N, Trauer S, Patzelt A, et al. Hair follicles contribute significantly to penetration through human skin only at times soon after application as a solvent deposited solid in man. Br J Clin Pharmacol. 201 1;72(5):768-74.

22. Otberg N, Patzelt A, Rasulev U, Hagemeister T, Linscheid M, Sinkgraven R, et al. The role of hair follicles in the percutaneous absorption of caffeine. Br J Clin Pharmacol. 2008;65(4):488-92.

23. Otberg N, Teichmann A, Rasuljev U, Sinkgraven R, Sterry W, Lademann J. Follicular penetration of topically applied caffeine via a shampoo formulation. Skin Pharmacol Physiol. 2007;20(4): 195-8.

24. Prow TW, Grice JE, Lin LL, Faye R, Butler M, Becker W, et al. Nanoparticles and microparticles for skin drug delivery. Adv Drug Deliv Rev Elsevier BV. 201 1;63(6):470-91.

25. Ngo MA, O’Malley M, Maibach HI. Percutaneous absorption and exposure assessment of pesticides. J Appl Toxicol. 2010;30(2):91-114.

26. Shamma RN, Aburahma MH. Follicular delivery of spironolactone via nanostructured lipid carriers for management of alopecia. Int J Nanomedicine. 2014;9(1):5449-60.

27. Lademann J, Otberg N, Richter H, Weigmann HJ, Lindemann U, Schaefer $\mathrm{H}$, et al. Investigation of follicular penetration of topically applied substances. Skin Pharmacol Appl Ski Physiol. 2001;14(suppl. 1):17-22.

28. Grams YY, Alaruikka S, Lashley L, Caussin J, Whitehead L, Bouwstra JA. Permeant lipophilicity and vehicle composition influence on accumulation of dyes in hair follicles of human skin. Eur J Pharm Sci. 2003;18(5):329-36.

29. Ylva Y, Grams JAB. Penetration and distribution of three lipophilic probes in vitro in human skin focusing on the hair follicle. J Control Release. 2002;83(2):253-62.

30. Meidan VM, Bonner MC, Michniak BB. Transfollicular drug delivery - is it a reality? Int J Pharm. 2005;306(1-2):1-14.

31. Bookout RL, Quinn DW, McDougal JN. Parallel dermal subcompartments for modeling chemical absorption. SAR QSAR Environ Res. 1997;7(1-4):259-79. 
32. Frum Y, Bonner MC, Eccleston GM, Meidan VM. The influence of drug partition coefficient on follicular penetration: in vitro human skin studies. Eur J Pharm Sci. 2007;30(3-4):280-7.

33. Domashenko A, Gupta S, Cotsarelis G. Efficient delivery of transgenes to human hair follicle progenitor cells using topical lipoplex. Nat Biotechnol. 2000;18(4):420-3.

34. Krause K, Foitzik K. Biology of the hair follicle: the basics. Semin Cutan Med Surg. 2006;25(1):2-10.

35. Valiveti S, Wesley J, Lu GW. Investigation of drug partition property in artificial sebum. Int J Pharm. 2008;346(1-2):10-6.

36. Valiveti S, Lu GW. Diffusion properties of model compounds in artificial sebum. Int J Pharm. 2007;345(1-2):88-94.

37. Lu GW, Valiveti S, Spence J, Zhuang C, Robosky L, Wade K, et al. Comparison of artificial sebum with human and hamster sebum samples. Int J Pharm. 2009;367(1-2):37-43.

38. Wang L, Chen L, Han L, Lian G. Kinetics and equilibrium of solute diffusion into human hair. Ann Biomed Eng. 2012;40(12): 2719-26.

39. Vogt A, Hadam S, Heiderhoff M, Audring H, Lademann J, Sterry W, et al. Morphometry of human terminal and vellus hair follicles. Exp Dermatol. 2007;16(11):946-50.

40. Robertson K, Rees JL. Variation in epidermal morphology in human skin at different body sites as measured by reflectance confocal microscopy. Acta Derm Venereol. 2010;90(4):368-73.

41. Mohd F, Todo H, Yoshimoto M, Yusuf E, Sugibayashi K. Contribution of the hair follicular pathway to Total skin permeation of topically applied and exposed chemicals. Pharmaceutics. 2016;8(4):32.

42. Chandrasekaran NC, Sanchez WY, Mohammed YH, Grice JE, Roberts MS, Barnard RT. Permeation of topically applied magnesium ions through human skin is facilitated by hair follicles. Magnes Res. 2016;29(2):35-42.

43. Patzelt A, Richter H, Buettemeyer R, Huber HJR, Blume-Peytavi U, Sterry W, et al. Differential stripping demonstrates a significant reduction of the hair follicle reservoir in vitro compared to in vivo. Eur J Pharm Biopharm. 2008;70(1):234-8.

44. Hindmarsh AC, Brown PN, Grant KE, Lee SL, Serban R, Shumaker DE, et al. SUNDIALS: suite of nonlinear and differential/algebraic equation solvers. ACM Trans Math Softw. 2005;31(3):363-96.

45. Sandby-Møller J, Poulsen T, Wulf HC. Epidermal thickness at different body sites: relationship to age, gender, pigmentation, blood content, skin type and smoking habits. Acta Derm Venereol. 2003;83(6):410-3.

46. Lee Y, Hwang K. Skin thickness of Korean adults. Surg Radiol Anat. 2002;24(3-4):183-9.

47. Zouboulis GC. Acne and sebaceous gland function. Clin Dermatol. 2004;22(5):360-6.

48. Aboagye B, Ahenkorah J, Hottor B, Addai F. Comparative characteristics of black and Grey chest and selected facial hairs in Negroid males. Internet J Biol Anthropol. 2014;7(1):1-9.

49. Otberg N, Richter H, Schaefer H, Blume-Peytavi U, Sterry W, Lademann J. Variations of hair follicle size and distribution in different body sites. J Invest Dermatol. 2004;122(1):14-9.

50. Mitragotri S. A theoretical analysis of permeation of small hydrophobic solutes across the stratum corneum based on scaled particle theory. J Pharm Sci. 2002;91(3):744-52.

51. Brachtel D, Richter E. Absolute bioavailability of caffeine from a tablet formulation. J Hepatol. 1992;16(3):385.

52. Mitragotri S, Johnson ME, Blankschtein D, Langer R. An analysis of the size selectivity of solute partitioning, diffusion, and permeation across lipid bilayers. Biophys J. 1999;77:1268-83.

53. Jackson GW, James DF. The permeability of fibrous porous media. Can J Chem Eng. 1986;64(3):364-74.

54. Kasting GB, Barai ND, Wang T-F, Nitsche JM. Mobility of water in human stratum corneum. J Pharm Sci. 2003;92(11):2326-40.

55. Guyton AGHJ. Textbook of medical physiology. 11th ed. Philadelphia: Elsevier; 2016.

56. Bookout RL Jr, McDaniel CR, Quinn DWMJ. Multilayered dermal Subcompartments for modeling chemical absorption. SAR QSAR Environ Res. 1996;5(3):133-50.

57. Einstein A. On the movement of small particles suspended in stationary liquids required by the molecular-kinetic theory of heat. Ann Phys. 1905;322:549-60.

58. Wang L, Chen L, Lian G, Han L. Determination of partition and binding properties of solutes to stratum corneum. Int J Pharm. 2010;398(1-2):114-22.

59. Kretsos K, Miller MA, Zamora-Estrada G, Kasting GB. Partitioning, diffusivity and clearance of skin permeants in mammalian dermis. Int J Pharm. 2008;346(1-2):64-79.

60. Ibrahim R, Nitsche JM, Kasting GB. Dermal clearance model for epidermal bioavailability calculations. J Pharm Sci. 2012;101(6): 2094-108. 